\title{
Research Article \\ Consensus of Discrete Time Second-Order Multiagent Systems with Time Delay
}

\author{
Wei Zhu \\ Research Center of System Theory and Application, Chongqing University of \\ Posts and Telecommunications, Chongqing 400065, China \\ Correspondence should be addressed to Wei Zhu, zhuwei@cqupt.edu.cn \\ Received 1 June 2012; Accepted 14 November 2012 \\ Academic Editor: Antonia Vecchio \\ Copyright (C) 2012 Wei Zhu. This is an open access article distributed under the Creative Commons \\ Attribution License, which permits unrestricted use, distribution, and reproduction in any \\ medium, provided the original work is properly cited. \\ The consensus problem for discrete time second-order multiagent systems with time delay is \\ studied. Some effective methods are presented to deal with consensus problems in discrete time \\ multiagent systems. A necessary and sufficient condition is established to ensure consensus. The \\ convergence rate for reaching consensus is also estimated. It is shown that arbitrary bounded time \\ delay can safely be tolerated. An example is presented to illustrate the theoretical result.
}

\section{Introduction}

The study of information flow and interaction among multiple agents in a group plays an important role in understanding the coordinated movements of these agents. As a result, a critical problem for coordinated control is to design appropriate protocols and algorithms such that the group of agents can reach consensus on the shared information in the presence of limited and unreliable information exchange as well as communication time delays.

In multiagent systems, communication time delays between agents are inevitable due to various reasons. For instance, they may be caused by finite signal transmission speeds, traffic congestions, packet losses, and inaccurate sensor measurements. In addition, in practical engineering applications, the agents in multiagent systems transmit sampled information by using sensors or communication network, and the coordination control algorithms are proposed based on the discrete time sampled data to achieve the whole control object. The typical discrete-time consensus control strategy was provided by Jadbabaie et al. [1], which is a simplified Vicsek model [2]. Recently, the consensus analysis of the discrete time 
first order multiagent systems with or without communication time delays has been studied extensively, see [3-6], to name a few. While it has been realized that modeling more complex practical processes needs the use of double integrator dynamics, as a result, cooperative control for multiple agents with double-integrator dynamics has become an active area of research. Compared with the first-order consensus, Ren and Atkins [7] show that the existence of a directed spanning tree is a necessary rather than a sufficient condition to reach second-order consensus. Therefore, the extension of consensus algorithms from first order to second order is nontrivial. In recent years, more attention has been paid to the consensus problem of multiagent systems with continuous time second-order systems and much progress has been made, some important works include [8-13]. But there has been little attention to the consensus of discrete time second-order systems. In [14], Lin and Jia investigate the consensus of discrete time second order multiagent systems with nonuniform time delays and dynamically changing topologies. A linear consensus protocol is introduced to realize local control strategies for these second-order discrete-time agents. In [15], by using the generalized Nyquist criterion and the Gerschgorin disc theorem, the consensus algorithm with a static leader is proposed to solve the consensus problem of the discrete time second-order multiagent systems with communication delays. In [16], the mean square consentability problem for a network of double-integrator agents with stochastic switching topology is studied. An LMI approach to the design of the consensus protocol is presented. Hence, the consensus problem for discrete time second-order multiagent systems is more important and challenging. The problem becomes more complicated when consensus protocols are extended to systems with time delay.

Motivated by above discussion, in this paper, we consider the consensus problems for discrete time second-order multiagent systems with time delay and provide some effective methods to deal with consensus problems in discrete time multiagent systems.

\section{Problem Statement}

Let $\{i \mid i \in \mathcal{U}\}$ be a set of $n$ agents, where $\mathcal{U}=\{1,2, \ldots, n\}$. A directed graph $\mathcal{G}=(\mathcal{U}, \boldsymbol{\varepsilon})$ will be used to model the interaction topology among these agents. The $i$ th vertex represents the $i$ th agents $i$. The set of out-neighbors of vertex $i$ is denoted by $\mathcal{N}_{i}=\{j \in \mathcal{V}:(i, j) \in \mathcal{E}\}$. A path in a digraph is a sequence $i_{0}, i_{1}, \ldots, i_{l}$ of distinct nodes such that $\left(i_{l_{1}-1}, i_{l_{1}}\right) \in \mathcal{E}, l_{1}=1,2, \ldots, l$. If there exists a path from node $i$ to node $j$, we say that $j$ is reachable from $i$. If $j$ is reachable from all other agents, $j$ is said to be globally reachable. A directed tree is a digraph, where every node has exactly one parent except for one node, called the root, which has no parent, and the root has a directed path to every other node. A directed spanning tree of a digraph is a directed tree formed by graph edges that connect all the nodes of the graph. We say that a graph has (or contains) a directed spanning tree if there exists a directed spanning tree that is a subset of the graph. In a digraph $\mathcal{G}$, if $\mathcal{U}$ is a nonempty subset of $\mathcal{U}$ and $u \nrightarrow v$ for all $u \in \mathcal{U}$ and $v \in \mathcal{V}-\boldsymbol{U}$, then $\boldsymbol{U}$ is said to be closed.

$A=\left(a_{i j}\right)_{n \times n}$ is the adjacency matrix, where $a_{i j}$ denotes the weight of edge $(i, j)$ and $a_{i j}>0$ if and only if $(i, j) \in \mathcal{E}$. Moreover, we assume that $a_{i i}>0$ for $i=1, \ldots, n$, that is, every agent can use its own instantaneous state information, the same assumption is also taken by [17]. Diagonal matrix $D=\operatorname{diag}\left\{d_{1}, d_{2}, \ldots, d_{n}\right\}$ is the degree matrix whose diagonal elements are defined by $d_{i}=\sum_{j \in \mathcal{N}_{i}} a_{i j}$. 
The dynamics of agent $i$ is described by

$$
\begin{aligned}
& x_{i}(t+h)=x_{i}(t)+v_{i}(t) h, \\
& v_{i}(t+h)=v_{i}(t)+u_{i}(t) h,
\end{aligned}
$$

where the update time instants $t \in R$ will be the form $t=t_{0}+p h, t_{0}$ is the initial moment, $p=1,2, \ldots$, the positive real number $h$ is the sampled time or time discretization unit, in this paper, we assume that $0<h<1 . x_{i}, v_{i}, u_{i}:[0, \infty) \rightarrow R, i=1,2, \ldots, n$, denote the position (or angle), velocity (or angular velocity), and control of agent $i$, respectively.

Definition 2.1. Second-order consensus in the multiagent systems (2.1) is said to be achieved if for any initial conditions,

$$
\lim _{t \rightarrow \infty}\left\|x_{i}(t)-x_{j}(t)\right\|=0, \quad \lim _{t \rightarrow \infty}\left\|v_{i}(t)-v_{j}(t)\right\|=0, \quad i, j=1,2, \ldots, n .
$$

\section{Consensus Analysis}

To solve the consensus problem, we introduce the following neighbor-based feedback control protocol

$$
u_{i}(t)=-v_{i}(t)-k \frac{1}{\sum_{j \in \mathcal{N}_{i}} a_{i j}}\left\{\sum_{j \in \mathcal{N}_{i}} a_{i j}\left[x_{i}(t)-x_{j}(t-\tau)+v_{i}(t)-v_{j}(t-\tau)\right]\right\}, \quad i=1,2, \ldots, n,
$$

where $k>0$ is a control parameter, $\tau \geq 0$ is the time delay.

Theorem 3.1. Under control protocol (3.1), for any bounded time-delay, there exist some $k>0$ such that the consensus for (2.1) is reached asymptotically if and only if the interconnection graph $\mathcal{G}$ of $n$ agents has a globally reachable node.

Before proving Theorem 3.1, we first need to do model transformation on systems (2.1) under control protocol (3.1) and give some technical lemmas.

Let $x(t)=\left(x_{1}(t), \ldots, x_{n}(t)\right)^{T}, v(t)=\left(v_{1}(t), \ldots, v_{n}(t)\right)^{T}, B=\left(b_{i j}\right)_{n \times n}$, where $b_{i j}=$ $a_{i j} / \sum_{j \in \mathcal{N}_{i}} a_{i j}$, then by (2.1) and (3.1), we have

$$
\begin{gathered}
x(t+h)=x(t)+v(t) h, \\
v(t+h)=-k h x(t)+(1-h-k h) v(t)+k h B x(t-\tau)+k h B v(t-\tau) .
\end{gathered}
$$

Since there has a globally reachable node in graph $\mathcal{G}$, without loss of generality, we assume the $n$th node is the globally reachable and set:

$$
Q=\left(\begin{array}{cc}
I_{n-1} & -1_{n-1} \\
0 & 1
\end{array}\right)
$$


Let $\tilde{x}(t)=Q x(t), \tilde{v}(t)=Q v(t)$, then $\tilde{x}(t)=\left(x_{1}(t)-x_{n}(t), x_{2}(t)-x_{n}(t), \ldots, x_{n-1}(t)-\right.$ $\left.x_{n}(t), x_{n}(t)\right)^{T}, \widetilde{v}(t)=\left(v_{1}(t)-v_{n}(t), v_{2}(t)-v_{n}(t), \ldots, v_{n-1}(t)-v_{n}(t), v_{n}(t)\right)^{T}$ and

$$
\begin{aligned}
\tilde{x}(t+h)= & \tilde{x}(t)+\tilde{v}(t) h, \\
\tilde{v}(t+h)= & -k h \tilde{x}(t)+(1-h-k h) \tilde{v}(t) \\
& +k h Q B Q^{-1} \tilde{x}(t-\tau)+k h Q B Q^{-1} \widetilde{v}(t-\tau) .
\end{aligned}
$$

Define $y_{1}(t)=\left(x_{1}(t)-x_{n}(t), x_{2}(t)-x_{n}(t), \ldots, x_{n-1}(t)-x_{n}(t)\right)^{T}, y_{2}(t)=x_{n}(t), z_{1}(t)=$ $\left(v_{1}(t)-v_{n}(t), v_{2}(t)-v_{n}(t), \ldots, v_{n-1}(t)-v_{n}(t)\right)^{T}, z_{2}(t)=v_{n}(t)$.

Noticing that the every row sum of $B$ is 1 , we have

$$
Q B Q^{-1}=\left(\begin{array}{cc}
C & 0 \\
B_{n}^{r} & 1
\end{array}\right)
$$

where $C=B_{n-1}-1_{n-1} B_{n}^{r}, B_{n-1}$ is a $(n-1) \times(n-1)$ matrix formed by the first $n-1$ rows and the first $n-1$ columns of matrix $B, B_{n}^{r}$ is a row vector formed by the first $n-1$ elements of the $n$th row of matrix $B$.

Then (3.3) can be decoupled as follows:

$$
\begin{aligned}
y_{1}(t+h)= & y_{1}(t)+z_{1}(t) h \\
z_{1}(t+h)= & -k h y_{1}(t)+(1-h-k h) z_{1}(t) \\
& +k h C y_{1}(t-\tau)+k h C z_{1}(t-\tau) .
\end{aligned}
$$

Let $\varepsilon(t)=\left(y_{1}^{T}(t), z_{1}^{T}(t)\right)^{T}$, one can obtain that

$$
\varepsilon(t+h)=H \varepsilon(t)+k h P \varepsilon(t-\tau), \quad t \geq t_{0}
$$

where

$$
H=\left(\begin{array}{cc}
I_{n-1} & h I_{n-1} \\
-k h I_{n-1} & (1-h-k h) I_{n-1}
\end{array}\right), \quad P=\left(\begin{array}{ll}
0 & 0 \\
C & C
\end{array}\right)
$$

Therefore, the consensus of (2.1) is achieved if and only if $\varepsilon(t) \rightarrow 0$ as $t \rightarrow \infty$ for any initial condition $\varepsilon(t)=\phi(t), t \in\left[t_{0}-\tau, t_{0}\right]$.

Now, we give some useful lemmas for proving Theorem 3.1.

Lemma 3.2 (see [18]). If a nonnegative matrix $A$ has the same positive constant row sums given by $\mu>0$, then $\mu$ is an eigenvalue of $A$ with an associated eigenvector 1 and $\rho(A)=\mu$, where $\rho(A)$ denotes the spectral radius. In addition, the eigenvalue $\mu$ of $A$ has algebraic multiplicity equal to one, if and only if the graph associated with A has a spanning tree.

Lemma 3.3. Equation (3.7) has a unique equilibrium 0 if the interconnection graph $\mathcal{G}$ of $n$ agents has a globally reachable node. 
Proof. It suffices to verify that $\left(I_{2(n-1)}-H-k h P\right) \varepsilon=0$, that is,

$$
\left(\begin{array}{cc}
0 & -h I_{n-1} \\
k h\left(I_{n-1}-C\right) & h I_{n-1}+k h\left(I_{n-1}-C\right)
\end{array}\right)\left(\begin{array}{l}
y_{1} \\
z_{1}
\end{array}\right)=0
$$

has a unique solution 0 if interconnection graph $\mathcal{G}$ has a globally reachable node.

By (3.9), it is obvious that $z_{1}=0$, then it is equivalent to prove that $\left(I_{n-1}-C\right) y_{1}=0$ has a unique solution $y_{1}=0$, namely, $C$ has no eigenvalue 1 .

Since the graph $\mathcal{G}$ associated with $A$ has a globally reachable node, by the definition of $B$, we know that the graph associated with $B^{T}$ has a directed spanning tree. By Lemma 3.2, matrix $B^{T}$ has the eigenvalue 1 with algebraic multiplicity 1 . Therefore, by (3.5), matrix $C^{T}$ has no eigenvalue 1 , that is, 1 is not the eigenvalue of matrix $C$. The proof is completed.

Lemma 3.4. For $0<h<1$, if $0<k<1$, then $\rho(H)<1$, where $\rho(H)$ represents the spectral radius of matrix $H$.

Proof. Let $\lambda$ be any eigenvalue of $H$, that is,

$$
\left|\lambda I_{2 n-2}-H\right|=\left|\begin{array}{cc}
(\lambda-1) I_{n-1} & -h I_{n-1} \\
k h I_{n-1} & (\lambda-1+h+k h) I_{n-1}
\end{array}\right|=0
$$

Case I. If $\lambda=1$, then it follows from the Laplace theorem for a partitioned matrix that $\operatorname{det}\left(k h I_{n-1}\right) \cdot \operatorname{det}\left(h I_{n-1}\right)=0$, that is $k h^{2}=0$, which is a contradiction.

Case II. For $\lambda \neq 1$, using the Laplace theorem for a partitioned matrix again, one can derive from (3.10) that $\operatorname{det}\left((\lambda-1) I_{n-1}\right) \cdot \operatorname{det}\left((\lambda-1+h+k h) I_{n-1}+\left(k h^{2} /(\lambda-1)\right) I_{n-1}\right)=0$, then

$$
\lambda^{2}+(k h+h-2) \lambda+k h^{2}-k h-h+1=0 .
$$

Therefore, we have $\Delta=(k h+h-2)^{2}-4\left(k h^{2}-k h-h+1\right)=h^{2}(k-1)^{2}$ and $\lambda_{1,2}=(2-h-k h \pm$ $\sqrt{\Delta}) / 2=(2-h-k h \pm h(1-k)) / 2$. One can easily verify that $\left|\lambda_{1,2}\right|<1$, that is, $\rho(H)<1$, for $0<h<1,0<k<1$. The proof is completed.

Lemma 3.5. If $\rho(H)<1$, then there exist positive constants $K \geq 1$ and $0<\gamma<1$ such that $\|H\|^{t-t_{0}} \leq K \gamma^{t-t_{0}}, t \geq t_{0}$.

Lemma 3.6. Inequality $x^{\tau+h}-\gamma x^{\tau}-l>0$ has at least one solution $x \in(\gamma, 1)$ if $1-\gamma-l>0$.

Proof. Let $f(x)=x^{\tau+h}-\gamma x^{\tau}-l$, then $f^{\prime}(x)=x^{\tau-1}\left((\tau+h) x^{h}-\tau \gamma\right)$. Set $g(x)=(\tau+h) x^{h}-\tau \gamma$, then $g^{\prime}(x)=h(\tau+h) x^{h-1}>0$, so $g(x)>g(\gamma)=(\tau+h) \gamma^{h}-\tau \gamma>0$ for $x \in(\gamma, 1)$. Thus $f(x)$ is monotonically increasing for $x \in(\gamma, 1)$. Since $f(1)>0$, so there exists at least a $x \in(\gamma, 1)$ such that $x^{\tau+h}-\gamma x^{\tau}-l>0$.

Lemma 3.7 (see [19]). A digraph $\mathcal{G}=(\mho, \mathcal{E})$ with $|\mho| \geq 2$ has no globally reachable node if and only if it has at least two disjoint closed subsets of $\mho$.

Now, we are in the position to prove Theorem 3.1. 
Proof. Sufficiency. By (3.5), one derives that

$$
\varepsilon(t)=H^{p} \varepsilon\left(t_{0}\right)+k h \sum_{s=0}^{p-1} H^{p-1-s} P \varepsilon\left(t_{0}+s h-\tau\right),
$$

where $t=p h+t_{0}, p=1,2, \ldots$

For $0<k<1$, by Lemma 3.4, $\rho(H)<1$. Noticing that Lemma 3.5, there exist constants $0<\gamma<1$ and $K \geq 1$ such that $\|H\|^{t-t_{0}} \leq K \gamma^{t-t_{0}}, t \geq t_{0}$. Therefore by (3.12), we have

$$
\|\varepsilon(t)\| \leq K \gamma^{p}\left\|\varepsilon\left(t_{0}\right)\right\|+k h \sum_{s=0}^{p-1} K \gamma^{p-1-s}\|P\| \cdot\left\|\varepsilon\left(t_{0}+s h-\tau\right)\right\| .
$$

For $k<(1-\gamma) / h K\|P\|$, by Lemma 3.6, there exists a positive constant $\lambda$ satisfying $\gamma<\lambda<1$ such that $\lambda^{\tau+h}-\gamma \lambda^{\tau}-k h K\|P\|>0$.

In the following, we will show that

$$
\|\varepsilon(t)\| \leq K \| \phi|| \lambda^{t-t_{0}}, \quad t \geq t_{0}
$$

where $\|\phi\|=\sup _{t \in\left[t_{0}-\tau, t_{0}\right]}\|\varepsilon(t)\|$.

It is clear that $\|\varepsilon(t)\| \leq K\|\phi\| \lambda^{t-t_{0}}$, for $t \in\left[t_{0}-\tau, t_{0}\right]$.

Next, we first show for any $\eta>1$,

$$
\|\varepsilon(t)\|<\eta K\|\phi\| \lambda^{t-t_{0}} \triangleq \varphi(t), \quad t \geq t_{0} .
$$

If (3.15) is not true, then there must exists a $t^{*}=t_{0}+p^{*} h\left(p^{*}>0\right)$ such that

$$
\|\varepsilon(t)\|<\varphi(t), \quad \text { for } t \in\left[0, t^{*}\right),\left\|\varepsilon\left(t^{*}\right)\right\|=\varphi\left(t^{*}\right)
$$

By (3.13), one can obtain that

$$
\begin{aligned}
\varphi\left(t^{*}\right)=\left\|\varepsilon\left(t^{*}\right)\right\| & \leq K \gamma^{p^{*}}\left\|\varepsilon\left(t_{0}\right)\right\|+k h \sum_{s=0}^{p^{*}-1} K \gamma^{p^{*}-1-s}\|P\| \cdot\left\|\varepsilon\left(t_{0}+s h-\tau\right)\right\| \\
& <K \eta\|\phi\| \gamma^{p^{*}}\left[1+\frac{k h K\|P\|^{p^{*}-1}}{\gamma \lambda^{\tau}} \sum_{s=0}\left(\frac{\lambda^{h}}{\gamma}\right)^{s}\right] \\
& \leq K \eta\|\phi\|\left[\gamma^{p^{*}}+\frac{k h K\|P\|}{\left(\lambda^{h}-\gamma\right) \lambda^{\tau}}\left(\lambda^{p^{*} h}-\gamma^{p^{*}}\right)\right] \\
& <K \eta\|\phi\| \lambda^{t^{*}-t_{0}}=\varphi\left(t^{*}\right),
\end{aligned}
$$

which is a contradiction. Thus, for any $\eta>1,(3.15)$ holds, let $\eta \rightarrow 1,(3.14)$ holds. Since there has a globally reachable node in graph $\mathcal{G}$, for $0<k<\min \{1,(1-\gamma) / h K\|P\|\},\|\varepsilon(t)\| \rightarrow 0$ as 


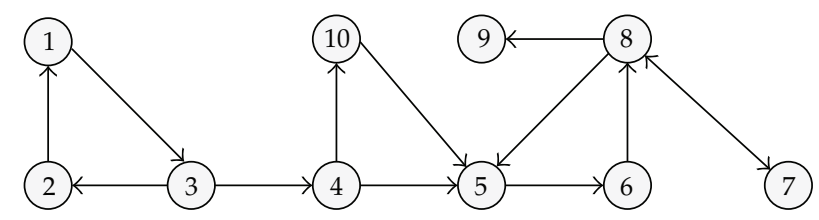

Figure 1: The directed interaction topology of ten agents.

$t \rightarrow \infty$ for any initial condition invoking Lemma 3.3, that is, there exist some $k>0$ such that the consensus for (2.1) is reached asymptotically.

Necessity. The consensus of (2.1) can be reached asymptotically, that is, for any initial position and velocity and any bounded time delay $\tau \geq 0, x_{i} \rightarrow x_{j}$ and $v_{i} \rightarrow v_{j}$ as $t \rightarrow \infty$. By way of contradiction, suppose that the graph $\mathcal{G}$ has no globally reachable node. Then it follows from Lemma 3.7 that there are at least two disjoint closed sets of nodes in graph $\mathcal{G}$. Without loss of generality, we consider the following special case, that is, $\tau=0$ and there are exactly two disjoint closed sets in graph $\mathcal{G}$, say $\boldsymbol{V}_{1}=\{1\}$ and $\boldsymbol{V}_{2}=\{2\}$, that is, there is only one node in $\boldsymbol{V}_{1}$ and $\boldsymbol{V}_{2}$, respectively, (if there are more disjoint closed sets or there are more nodes in each disjoint closed sets, it can be proved by a similar argument only with more complex computation). Given the initial condition satisfying $x_{1}\left(t_{0}\right)=v_{1}\left(t_{0}\right)=c_{1}$ and $x_{2}\left(t_{0}\right)=v_{2}\left(t_{0}\right)=$ $c_{2}$, by a direct computation, we have $x_{1}(t)=\left(2-(1-h)^{p}\right) c_{1}, v_{1}(t)=(1-h)^{p} c_{1}, x_{2}(t)=$ $\left(2-(1-h)^{p}\right) c_{2}, v_{2}(t)=(1-h)^{p} c_{2}$, where $t=t_{0}+p h, p=1,2, \ldots$. Hence, if $c_{1} \neq c_{2}$, the consensus cannot be reached, a contradiction. The proof of Theorem 3.1 is completed.

Remark 3.8. By the proof procedure of Lemma 3.4 and Theorem 3.1, one can conclude that Lemma 3.4 and Theorem 3.1 are also true for $h=1$. When $h=1$ and there is no communication time delay between agents, that is, $\tau=0$, Theorem 3.1 is consist with Theorem 2 in [16].

\section{A Simulation Example}

In this section, an example is given to demonstrate the efficiency and applicability of the proposed method and to validate the theoretical analysis. For simplicity, we suppose that all the edge weights are 1 in the following example.

Example 4.1. Assume that the interaction digraph of ten agents is depicted in Figure 1.

A globally reachable node can be easily found in the digraph. The initial positions and velocities of the ten agents are chosen as $[\sin (t), 2 \sin (t), \cos (t), t, 2 t, 2 \cos (t), 3 \sin (t)$, $4 \sin (t),-\sin (t),-\cos (t)]^{T}$ and $\left[\cos (t), t, t^{2}, \sin (t), \sin (2 t),-\cos (t), 2 \sin (t), 2 \cos (t), 3 \sin (t)\right.$, $3 \cos (t)]^{T}, t \in[-\tau, 0]$, respectively. Select control parameter $k=0.5$, transmission time delay $\tau=1$, and $h=0.01$. The simulation result under the control protocol (3.1) is shown in Figure 2, which illustrates that consensus has been achieved within about 150 seconds. Change transmission time delay as $\tau=0.5,1.5,2$, and 10 , by simulation experiments, we also find that consensus is accomplished within about 120,200, 250, and 700 seconds, respectively. This shows that our designed algorithms can effectively tolerate arbitrary bounded time delays. But when the time delay increased, the convergence rate will decrease. 


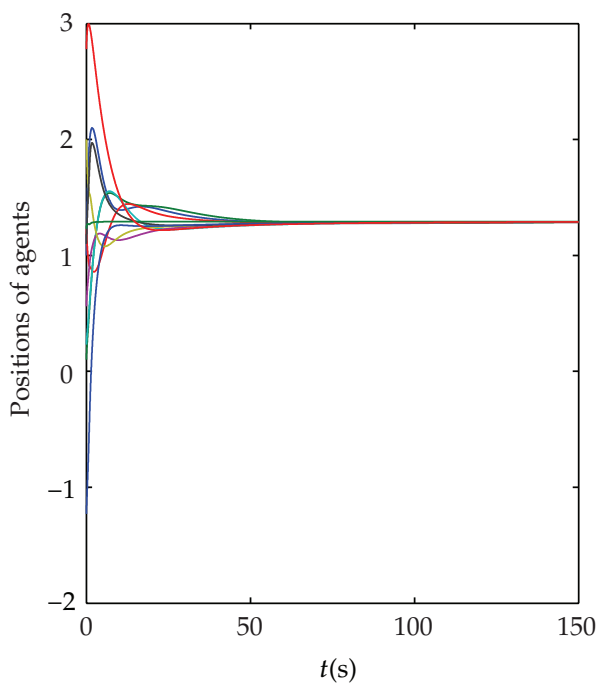

(a)

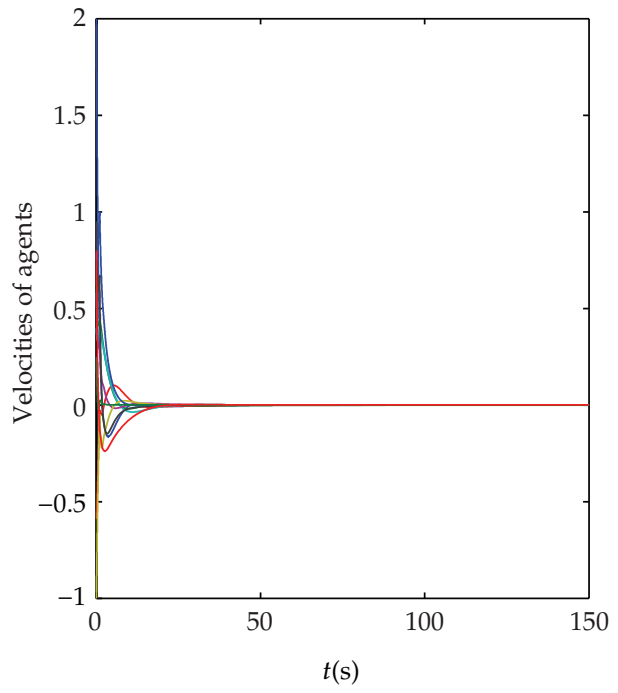

(b)

Figure 2: Positions and velocities of ten agents under control (3.1).

\section{Conclusion}

Based on algebraic graph theory, matrix theory, and stability theory of difference equation, the consensus problem of discrete time second-order multiagent systems with time delay is investigated. A necessary and sufficient condition for achieving consensus is presented. Furthermore, the convergence rate for consensus is given. The main results presented in this work are delay-independent (i.e., the results are valid for arbitrary bounded time delay). In addition, the present paper applies graph theoretic tools to explore explicit graphical conditions of the information exchange topologies under which consensus can be achieved. Since the interagent connection structures may vary over time, the consensus of discrete time second-order multiagent systems with time delays and switching topologies is also very interesting to us; this case will be investigated in future research.

\section{Acknowledgments}

The work is supported jointly by National Natural Science Foundation of China under Grant 61004042, Construction Project of Engineering and Technology Research Center of Chongqing (cstc2011pt-gc40006), and Foundation of Science and Technology project of Chongqing Education Commission under Grant KJ100513.

\section{References}

[1] A. Jadbabaie, J. Lin, and A. S. Morse, "Coordination of groups of mobile autonomous agents using nearest neighbor rules," IEEE Transactions on Automatic Control, vol. 48, no. 6, pp. 988-1001, 2003.

[2] T. Vicsek, A. Czirók, E. Ben-Jacob, I. Cohen, and O. Shochet, "Novel type of phase transition in a system of self-driven particles," Physical Review Letters, vol. 75, no. 6, pp. 1226-1229, 1995. 
[3] L. Moreau, "Stability of multiagent systems with time-dependent communication links," IEEE Transactions on Automatic Control, vol. 50, no. 2, pp. 169-182, 2005.

[4] L. Wang and F. Xiao, "A new approach to consensus problems in discrete-time multiagent systems with time-delays," Science in China F, vol. 50, no. 4, pp. 625-635, 2007.

[5] F. Xiao, L. Wang, and A. Wang, "Consensus problems in discrete-time multiagent systems with fixed topology," Journal of Mathematical Analysis and Applications, vol. 322, no. 2, pp. 587-598, 2006.

[6] F. Xiao and L. Wang, "Consensus protocols for discrete-time multi-agent systems with time-varying delays," Automatica, vol. 44, no. 10, pp. 2577-2582, 2008.

[7] W. Ren and E. Atkins, "Distributed multi-vehicle coordinated control via local information exchange," International Journal of Robust and Nonlinear Control, vol. 17, no. 10-11, pp. 1002-1033, 2007.

[8] P. Lin and Y. Jia, "Consensus of a class of second-order multi-agent systems with time-delay and jointly-connected topologies," IEEE Transactions on Automatic Control, vol. 55, no. 3, pp. 778-784, 2010.

[9] W. Zhu and D. Cheng, "Leader-following consensus of second-order agents with multiple timevarying delays," Automatica, vol. 46, no. 12, pp. 1994-1999, 2010.

[10] J. Qin, H. Gao, and W. X. Zheng, "Second-order consensus for multi-agent systems with switching topology and communication delay," Systems \& Control Letters, vol. 60, no. 6, pp. 390-397, 2011.

[11] H. Su, G. Chen, X. Wang, and Z. Lin, "Adaptive second-order consensus of networked mobile agents with nonlinear dynamics," Automatica, vol. 47, no. 2, pp. 368-375, 2011.

[12] Y. G. Sun and L. Wang, "Consensus problems in networks of agents with double-integrator dynamics and time-varying delays," International Journal of Control, vol. 82, no. 10, pp. 1937-1945, 2009.

[13] W. Yu, G. Chen, and M. Cao, "Some necessary and sufficient conditions for second-order consensus in multi-agent dynamical systems," Automatica, vol. 46, no. 6, pp. 1089-1095, 2010.

[14] P. Lin and Y. Jia, "Consensus of second-order discrete-time multi-agent systems with nonuniform time-delays and dynamically changing topologies," Automatica, vol. 45, no. 9, pp. 2154-2158, 2009.

[15] D. J. Liu and C. L. Liu, "Consensus problem of discrete-time second-order multi-agent network with communication delays," in Proceedings of the 3rd International Symposium on Intelligent Information Technology Application (IITA '09), pp. 340-344, November 2009.

[16] Y. Zhang and Y.-P. Tian, "Consentability and protocol design of multi-agent systems with stochastic switching topology," Automatica, vol. 45, no. 5, pp. 1195-1201, 2009.

[17] D. Angeli and P.-A. Bliman, "Stability of leaderless discrete-time multi-agent systems," Mathematics of Control, Signals, and Systems, vol. 18, no. 4, pp. 293-322, 2006.

[18] W. Ren and R. W. Beard, "Consensus seeking in multiagent systems under dynamically changing interaction topologies," IEEE Transactions on Automatic Control, vol. 50, no. 5, pp. 655-661, 2005.

[19] Z. Lin, B. Francis, and M. Maggiore, "Necessary and sufficient graphical conditions for formation control of unicycles," IEEE Transactions on Automatic Control, vol. 50, no. 1, pp. 121-127, 2005. 


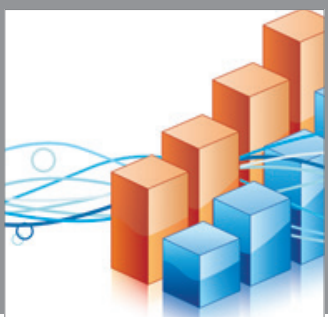

Advances in

Operations Research

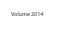

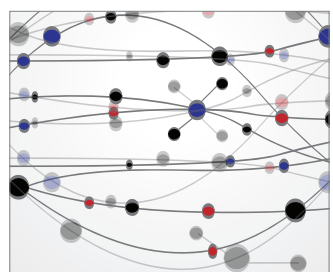

\section{The Scientific} World Journal
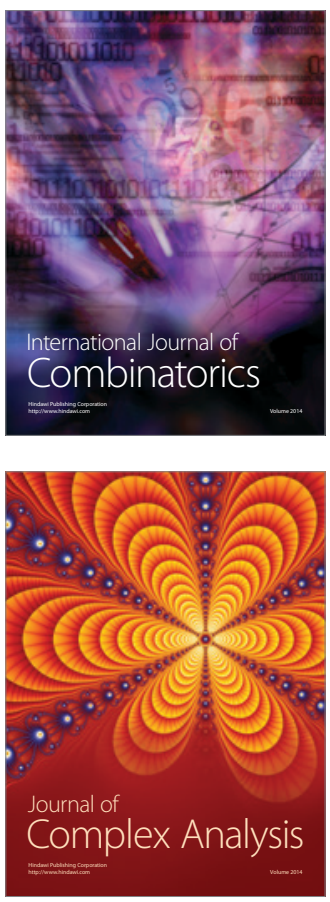

International Journal of

Mathematics and

Mathematical

Sciences
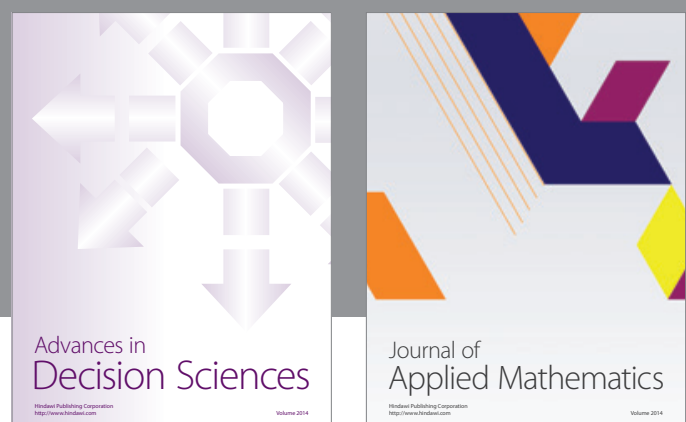

Journal of

Applied Mathematics
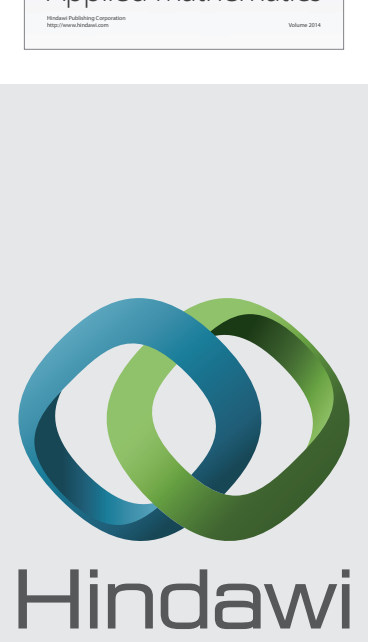

Submit your manuscripts at http://www.hindawi.com
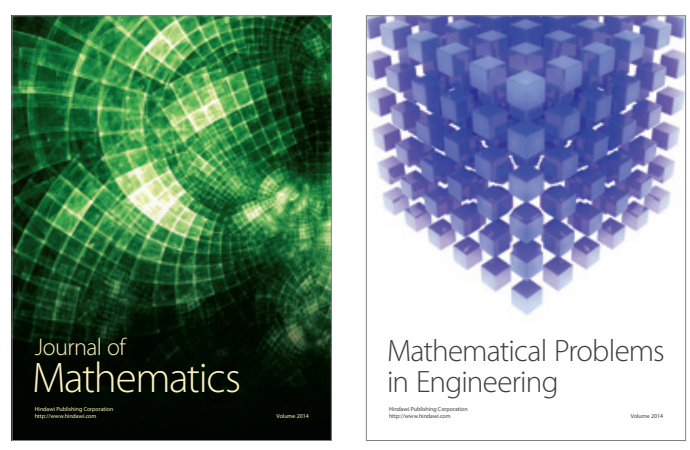

Mathematical Problems in Engineering
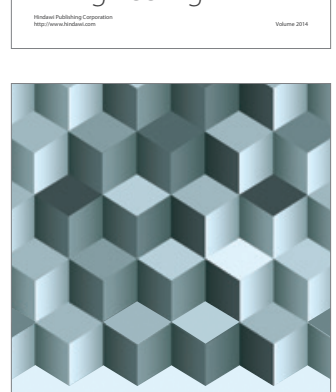

Journal of

Function Spaces
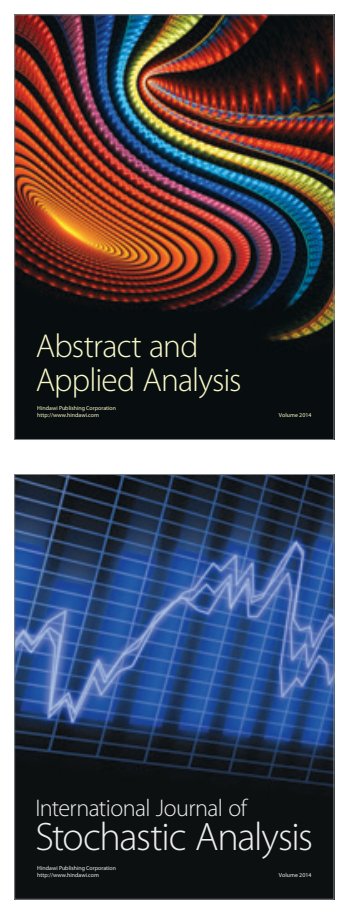

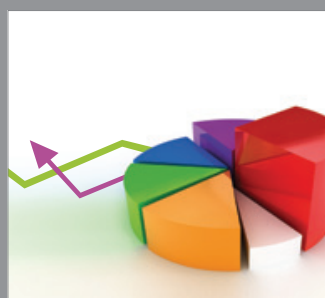

ournal of

Probability and Statistics

Promensencen
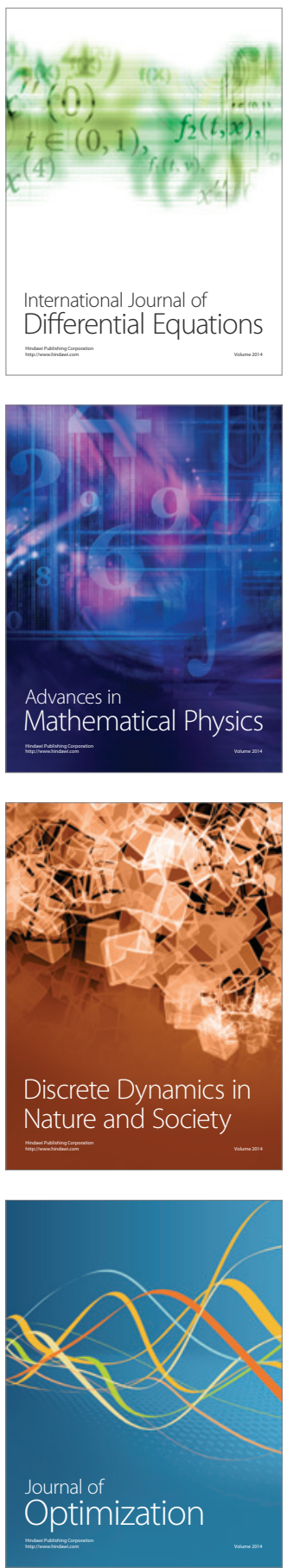